\title{
Using A Semiotic Metatheory for \\ Theory Understanding, Appraisal, and Use: \\ An Illustrative Social Work Translation of the \\ Affect Control Theory of Emotion
}

\author{
James A. Forte
}

\begin{abstract}
Charles Sanders Peirce's creed, "Do Not Block Inquiry," and his triadic model of the signs serve as the base for a semiotic metatheory of science and scientific theory. Semioticians characterize science as a universe of diverse sign systems, and scientists as members of different language communities. This paper introduces this approach. Affect control theorists ponder and investigate how actors, identities, actions, objects, emotions, and social settings are interrelated during interaction. Semiotic tools and principles guide the translation of the Affect Control Theory (ACT) of emotion. ACT is summarized and appraised for its value in increasing our understanding of human behavior in the social environment, its suitability to social work, and its applicability. ACT technical words are translated into simpler language, ACT displays into words, and ACT's interactionist language is translated into the language of ecosystems theory. Suggestions for strengthening ACT and for promoting semiotic translation are included.
\end{abstract}

Keywords: Semiotics, human behavior and the social environment, translation, theory

W

hat language should North American social workers speak? English, only. No, Bloom and others (1991) argued, convincingly, that competent social workers must achieve fluency in many additional languages. Besides our native language, professional practitioners must learn to converse in the language of clients, research, information management, social work practice (the profession's distinctive jargon), values, and theory. Often, these languages seem foreign and assistance is required. Social workers have developed strategies for translating the language of research into practice (Allen-Meares, Hudgins, Engberg, \& Lessnau, 2005). Translation research refers to "the practice of translating basic science data or discoveries from the laboratory bench into clinical applications aimed at treating various diseases" (Hudgins \& Allen-Meares, 2000, p. 2). Translation research bridges diverse professional arenas and specialized languages. The translator works

James A. Forte, MSW, Ph.D. is associate professor of Social Work at the Department of Social Work, Salisbury University, Salisbury, MD, 21801.

Copyright $^{\bullet} 2007$ Advances in Social Work Vol. 8 No. 1 (Spring 2007) 1-18.

Indiana University School of Social Work. 
to make empirical studies accessible and applicable by creating simple, short summaries, for instance.

Translation research lacks a metatheory of science and a methodology. Moreover, translation work has not been extended to the other languages identified by Bloom, Wood, and Chambon (1991). In this paper, I will concentrate on theory literacy and fluency and introduce a semiotic metatheory for theory translation and its distinctive vocabulary (Forte, 2001, 2002, 2006). I will illustrate the semiotic translation methodology by rendering the Affect Control Theory (ACT) of emotions into a language clear and useful for social work, appraising ACT's suitability for diverse practice challenges, and decoding the theory's implicit directives for application.

In this first section, I present the essentials of a semiotic approach to theory translation. Metatheorizing is an activity that involves theorizing about theorizing (Ritzer, 1991). This activity mandates "reflexivity," the self-examination of the profession's theoretical development by the profession's members (Zhao, 2001). Social work is a pragmatic profession that applies tested theories to ameliorate personal and public problems. Here, I develop a new type of metatheorizing for social work use: metatheorizing for application. The prefix "meta" means "above" or "beyond" (Zhao, 2001). Metatheorizing can result in the creation of a metatheory, a theory about theories. A metatheory's subject matter is the process of theorizing and the structure of the theory. The semiotic metatheory presented in this paper originates in the work of Charles Sanders Peirce. Peirce was the founder of semiotics, "the systematic investigation of the nature, properties, and kinds of signs, especially when undertaken in a self-conscious way" (Colapietro, 1993, p. 179). Peirce asserted, "everything may be comprehended or more strictly translated by something" (cited in Osimo, p. 606). Meaning, for Peirce is "the translation of a sign into another system of signs" (Osimo, 2002, p. 620). Peirce's semiotics was part of his philosophy of science commitment to the credo, "Do not block inquiry."

A sign is "anything that stands for something" (Colapietro, 1993, p. 180). There are three major types of signs. An icon "designates a type of sign in which the sign vehicle represents its object by virtue of a resemblance or similarity" (Colapietro, 1993, p. 114). Examples include a text, chart, diagram, and a map (Danesi, 1998). An index is a type of sign that represents the object by virtual or real connection; for instance, a weathervane indicates wind direction by connection to the wind (Colapietro, 1993). A symbol is "a sign based on convention or established usage or habit" (Colapietro, 1993, p. 190). A symbol stands for its referent in an arbitrary, conventional way (Danesi, 1998), as the word "tune-in" refers to an important helping action.

Semioticians conceive science to be a multimodal sign system. Metatheory or "metascience" is "the science of sciences," because of its attention to "the methods, history, sociology, and language of sciences" (Morris, 1946, p. 510). Morris, a systematizer of Peirce's intellectual system, added that social-humanistic sciences (referred to by social workers today as HBSE theories) "are semiotical sciences, in that the subject matter studied always involves sign processes and, correspondingly, the terms of the sciences always require for their definition reference to terms in the theory of signs" (p. 511). Scientists communicate by using linguistic sign systems and sign systems using diagrams, graphs, figures, gestures, statistics, and 
mathematical formulas (Sarukkai, 2002). Science is a sign system, but it is a sign system that is different in important ways from every day English. Scientific communication requires a precision, a degree of technical knowledge, a mastery of logical argument, a vocabulary, and a familiarity with notations that is not expected in everyday discourse (Halliday, 1978; Hodge \& Kress, 1988). Each theoretical tradition is a distinctive sign system with its own central metaphors, core concepts, categories, typical sentences, implicit grammatical rules, pronunciations, dialects, preferred displays, and particular network of native-speakers (Watson, 1985). Because of the multimodality of science and the complexity of its signs, social workers and their clients need assistance in decoding theories.

Translation is the act of translating from one sign system into another, rendering meanings intelligible across sign systems (Forte, 2002). Although Bloom (1975) did not recommend a semiotic metatheory for language mastery, he viewed translation as central to social work problem solving, "the major task of using theories from the literature is to find them ... to know how to understand them, and, finally, to translate them into direct strategy statements" (p. 162). In the remainder of this paper, I will translate the Affect Control Theory of emotion. My efforts will interweave three strategies (Jacobsen, 1965). Intralingual translation involves the interpretation of ACT as a sign system in its own theoretical language, symbolic interactionism. Interlingual translation is the interpretation of the ACT sign system in the native language of North American social workers, plain English. Intersemiotic translation involves the interpretation of ACT's nonverbal signs (displays, models, and formulas) into words, and the interpretation of ACT words as an eco-map, social work's preeminent visual sign system.

\section{TRANSLATION FOR UNDERSTANDING: HISTORICAL ROOTS}

Language translation requires first the placement in space and time of the sounds of the foreign speaker. Theory translation requires similar placement (Forte, 2002). ACT is located in the discipline of sociology, so that social structure and social situations are considered the sources of emotional experience, labeling, and expression. In the early 1980s, sociologists developed an informal network or subdiscipline called the "sociology of emotions," with its own association, newsletter, and conference workshops (Smith-Lovin, 1989, 1995). ACT affirms the sociology of emotions premises that emotions are linked to "aspects of social action including identity, role, identity, and culture" and "emotional responses are an integral part of social interaction" (Smith-Lovin, 1990, p. 238). ACT and Arlie Hochschild's emotion management theory have become the "cornerstones of this subdiscipline" (Lively \& Heise, 2004, p. 1110).

Affect Control Theory is located in the symbolic interactionist theoretical school. Affect control theorists share with all interactionists the conviction that the human experience is based in language. Heise (2007) claims, "your ability to interpret actions is built into the languages you speak. Languages everywhere provide nouns and verbs that describe actions in noun-verb sentences" (p. 35). He adds that people use language to describe events, actors, behaviors, emotions, objects, settings, and social actions in culturally standard ways. Affect control theorists share the interactionist aversion to dualistic thinking. The environment influences 
how a person experiences and manages emotions, but the person is active in creating definitions of the emotions and actions responsive to emotions (SmithLovin, 1995, p. 128). Identities are a function of both situational pressures and personal inclinations. Affect control theorists have an affinity for the structural symbolic interactionism. Members of this sub-school give attention to how society, through stable and reoccurring experiences in institutions and roles, structures the self, and the self then shapes behavior. Affect control theorists share the structuralist appreciation for quantitative research and experiments (Smith-Lovin, 2003). Affect control theorists consider role theory another kindred school of thought. Both assume that actors learn "a set of behavioral expectations associated with a social position" (Robinson \& Smith-Lovin, 2006, p. 137). Both agree that, when self and others conform to expectations, positive emotions result. When self and others deviate, negative emotions result. Other sociological theoretical languages have influenced affect control theorists (Francis, 2003). These include Stryker's Identity Theory, Goffman's Impression Management Theory, and Labeling Theory. Affect control theorists have borrowed ideas and words from psychology's attribution, balance, and consistency theories and from Powers' control model of perception (MacKinnon, 1994). Heise and O'Brien (1993) note a commonality with social constructionists who also conceive of "emotions as intelligent conduct, contrived according to cultural rules, so as to effect desired interpersonal outcomes" (p. 490).

While affect control theorists speak the language of interactionism, they reject the theoretical assumptions and language of other schools. Skinnerian behaviorists argue that there is no interpretation between stimuli and response; emotions are triggered automatically and the actor is not an active constructor of situations (Smith \& MiowLin, 2006). Parsonian functionalists argue that the person is completely socialized into playing scripted roles within stable institutions. The creative interpretation and use of emotions is minimal (Smith-Lovin \& Robinson, 2006). Psychodynamic theorists emphasize the unconscious, instinctual aspects of emotions and how shame and guilt are controlled through mechanisms of repression (Heise \& O'Brien, 1993). Organismic, bioevolutionary theorists view emotion as a byproduct, not an integral element, of interaction and focus on the equivalence between animals and humans even in emotional display (Smith-Lovin, 1995). ACT developers consider the sign systems of these theorists bizarre. Affect control theorists have organized a global network organized around a distinctive and shared "theoretical research program" (Heise, 2007). Numerous theorists collaborate in or at least communicate about theory expansion, research projects, and software development. The Affect Control Theory Internet Site (http://www.indiana.edu/ socpsy/ACT/), developed in 1997, posts 175 readings, software, conferences, and exhibits.

What are the historical origins of the theory as they have shaped theoretical language? Here, I will translate by use of exemplary role models (Forte, 2006). George Herbert Mead was the founder of symbolic interactionism. Mead theorized about language as the basis for a social psychology of mind, self, and society (MacKinnon, 1994.) People use culturally provided symbols to assign meaning to social situations and their elements (Robinson \& Smith-Lovin, 2006), and people 
escape the boundaries of individual consciousness to coordinate action via the use of shared symbols provided by the speech community. The person develops a self and adjusts behavior in membership groups by the feedback provided through taking others perspectives. ACT shares Mead's emphasis on language as a theoretical starting point and Mead's pioneering use of the root metaphor: the self as a cybernetic system. Act has also incorporated into its vocabulary many Meadian key words, including meaning, situation, definition of the situation, role, role taking, identity, self, and coordinated action. Mead's theorizing, however, was thoroughly cognitive, and he gave minimal attention to emotions. ACT advances symbolic interactionism by operationalizing its major concepts, including meaning, identity, behavior, and setting and by focusing on the affective meaning of objects. Unlike Mead, ACT assumes and documents with research evidence that affective meanings are more or less shared as cultural sentiments. Unlike Mead, ACT believes that actors communicate private emotional experience through emotional displays and narratives, and such communication evokes symmetry of responses similar to that in cognitive communication.

David R. Heise is the second major ACT exemplar (Smith-Lovin, 1999). A professor of sociology at Indiana University, he was the first to develop the control system perspective on identity. His statement in 1971 at the Southern Sociological Society meetings, explicated the major ACT tenets about identity, action, and meaning. He has also developed software to facilitate the analysis of social life, conceived of a methodology called event structure analysis, and nurtured many students. Most ACT "texts" considered in this paper were written or co-written by Heise, written by his students, or inspired by his example. David Heise was also the recipient of the Cooley-Mead Award for distinguished lasting career contribution to sociology.

How do the members of the contemporary theory community make use of their historical texts? Translation by intertextuality starts from the semiotic conviction that translation should trace "the allusion of a text to some other text" (Danesi, 1998, p. 286). Affect control theorists seem to agree on the tradition's classics (Francis, 2006; Heise, 2007; Smith-Lovin \& Robinson, 2006). In 1979, Heise published "Understanding Events." This first major book-length statement included the essential theoretical principles, mathematical formulas, measurement tools, and dictionaries. In 1987, Smith Lovin's article extended the actor-behavior-object predictive model to include settings. The ABOS model can thereby investigate settings, sentiments associated with a particular setting, such as a church, and how setting sentiments relate to other sentiments. Smith-Lovin and Heise (1988) next published Analyzing Social Interaction. This book includes empirical studies, theoretical elaborations, refinements of mathematical formulas, and reports on theory tests. The collection includes an article showing how the meaning of actors can be modified by social identities (membership characteristics, such as class, race, gender), dispositional traits (extroverted agency director), and mood and emotion descriptors (depressed client or angry father), and how such modifiers enrich the model (Averett \& Heise, 1987). In the late 1980s, there were a series of studies exploring emotions and their expression (MacKinnon, 1994), followed by a formal call for more emotion theorizing and research (Smith-Lovin, 1995). In 1994, 
MacKinnon published Symbolic Interactionism as Affect Control, a sophisticated theoretical systematization that organized the theory as seven sets of propositions. This represented a large-scale intersemiotic effort to translate ACT's mathematical models into verbal statements (Smith-Lovin \& Robinson, 2006). In 2002, Rashotte added nonverbal behavior into the event grammar (ABOs becomes ANBOS) and demonstrated how nonverbal behavior modifies the meaning of the behavior. Contemporary ACT researchers and theorists frequently cite these classics. Recently, Heise (2007) released Expressive Order, an intersemiotic and interlingual translation of ACT from mathematical and interactionist language into plain English. This may become the next classic.

\section{TRANSLATION FOR UNDERSTANDING: CURRENT DEVELOPMENTS}

In this section, I offer a translation of the contemporary set of ACT texts, with a focus on emotions and reference to significant work, conceptual developments, and empirical studies. ACT is a multimodal sign system. Its conception of the interrelation of the actor, identity, behavior, emotion, and setting is represented in visual form (displays and diagrams), lexical form (words, sentences, and paragraphs), and mathematical form (equations and formulas) (Scher \& Heise, 1993). I will prioritize the translation of words.

David Heise (2007) has worked to make his theory comprehensible. He provides intralingual translations (Symbolic Interactionism to plain English) and intrasemiotic translation (equations and formulas represented by mathematical symbols into plain English words). His summary of the 30 -year body of work provides "an accessible introduction to affect control theory for advanced undergraduates and graduates" and "communicates affect control theory conversationally, in words enriched with some figures and tables" (preface, 2007, p. i). For example, Heise (2007) clearly identifies focal questions asked by affect control theorists. What are expected behaviors for persons enacting various role identities? How do these behaviors change because of unusual events? What are the typical emotional reactions to victimization or oppression? How do emotions vary by identity, setting, and events? In a particular situation, what must one do to feel positive emotions, such as joy, and what negative emotions might be anticipated? How do identity, social interaction, and emotional response vary across cultures?

\section{Translation by Key Words and Dictionaries}

Theory understanding requires mastery of a theory's vocabulary. Translation by key words and dictionaries serve as important theory translation tools (Forte, 2001, 2006). Heise (2007) provides nominal definitions of 45 concepts central to ACT, a simple dictionary. Here are a few illustrations. Affect refers to "emotions, sentiments, impressions, and motives" (p. 145). Affective meaning is "the connotation of a word or symbol, measured as an evaluation-potency-activity (EPA) profile" (p. 145). Affect control theory focuses on "resisting changes in affective meanings and actualizing sentiments" (p. 145).

Affect control theorists include much definitional discussion in their publications. Several nominal definitions are focused on emotions. Affect control theory 
proposes, "humans try to manage experiences so that immediate feelings about people, actions, and settings affirm long term sentiments" (Heise, in MacKinnon, 1994, p. xi). Affect is "a general mode of consciousness pervading all our cognitions" (MacKinnon, 1994, p. 123). Affect includes "any evaluative (positive or negative) orientation toward an object, such as emotions like contentment and anger, attitudes like liking and disliking, and connotative meanings" (Smith-Lovin, 1995, p. 118). Emotions are "ephemeral affective experiences signaling the extent to which situational identities are being confirmed or disconfirmed in social interaction" (MacKinnon \& Goulborne, 2006, p. 245). A mood is an "emotional state of longer duration than emotion" (MacKinnon, 1994, p. 139). Emotion norms are "cultural norms that specify the type of emotion, the extent of emotion, and the duration of feeling that are appropriate in a situation" (Smith-Lovin, 1995, p. 124). Fundamental sentiments are "subconscious references that people have learned from their cultural heritage about the affective meanings about such everyday concepts as individual traits and states, the settings in which interactions take place, the identities of people involved in interactions, and behaviors of people in these interactions" (Francis, 2006. p. 139). Transitory impressions are the "moment-tomoment feelings about the identities and behaviors involved in the interactions, especially about the self" (Francis, p. 143). A deflection is "the discrepancy between a fundamental sentiment and a transient impression “(Turner \& Stets, 2006, p. 136).

\section{Translation by Architectonic Maps}

Theory understanding requires grasp of the language's grammar and how theoretical elements are organized into hierarchical order. Architectonic is a term adapted by Peirce to describe the systematic form a body of knowledge takes (Colapietro, 1993). Affect control theorists have diligently made explicit their assumptions. I will report those related to emotion. First, people react affectively to every event (MacKinnon, 1994). Second, the appraisal of an event, its categorization, or label, influences the affective impact of the event (Smith-Lovin, 1990), and appraisal considers three dimensions: evaluation, power, and activity. Third, people seek to create events that maintain consistency between fundamental sentiments and their moment-to-moment feelings (Francis, 2006). Fourth, people manage their emotions and conform to emotion norms by effecting culturally acceptable emotion displays (Heise \& O’Brien, 1993) and, fifth, emotions signal spontaneously the actor's success or failure in confirming fundamental sentiments and complying with norms (Francis, 2006). Finally, membership processes are critical to emotions. ACT "conceives of person in a context, examines how a society's culture and social structures influence the arousal, flow, and display of emotions and how people use emotions to constitute culture and maintain structures" (Turner \& Stets, 2006, pp. 1-2). ACT’s assumptive foundation differs from theoretical languages that assume emotions are primitive biological reflexes, indicative primarily of individual processes, independent of context, automatic, rather than constructed, and private (Francis, 2003; Heise \&Weir, 1999).

Pepper (1942), a philosopher, brought direction to the decoding of complicated theoretical architectonics. He argued that each framework of thought is built on a root metaphor. Affect control theorists rely on several root metaphors. Borrowing from Mead, the person is compared to a control system (Francis, 2006; Heise, 1979; 
MacKinnon, 1994). The person uses cybernetic processes, such as those in a house's cooling system. The person seeks to maintain an ideal state defined in terms of standards or reference settings stored in memory; fundamental sentiments are our thermostat settings. Like a cybernetic system, the person obtains input (perceptions and information) by monitoring the environment (situations). Fluctuations in perceptual signals (transitory impressions) are compared to reference settings (fundamental EPAs for the situational elements) to determine whether conditions are at variance with the system's standards. There is continuous comparison. If errors occur, the person takes restorative actions, similar to the thermostat activating the air conditioning to cool the house after a blast of hot air. The desired equilibrium is again realized; transient impressions are realigned with fundamental sentiments. In the human system, emotionality is the critical signaling process. Emotions signal the degree to which events confirm or disconfirm the actor's identity meanings as a good teacher, a trustworthy friend, or a competent social worker. Turner and Stets (2006) remark, "emotions are thus the gyroscopes of human behavior, keeping it on track in diverse situations, so that individuals can experience positive and avoid negative emotions" (p. 22).

Affect control theorists borrow interactionist imagery for their environment metaphor. A social environment is like a warehouse of symbols. It provides members with the names to designate all forms of social stimuli: identities, identity modifiers, and emotions. The warehouse contains dictionaries for cultural and subcultural groups. Each dictionary records the group's essential concepts and their meanings. These dictionaries accumulate across generations through members' experiences with others, public interactions, and the mass media. Different institutions, such as religion and academia, use different subsets of the society's dictionary: the nouns, adjectives, and verbs necessary for institutional action (Heise, 2007). From these dictionaries, individuals select relevant symbols to quickly orient themselves to a variety of situations. Affect control theorists inspired by this root metaphor have studied the United States and compiled its dictionary with EPA ratings for 500 social identities, 500 social behaviors, 300 emotion and trait terms, and 300 social settings (Francis, 2006).

Affect control theorists also compare the theoretical notion, "assignment of meaning," to a three-dimensional space. Meaning occurs, in this comparison, in a room containing all possible sentiments. Any particular EPA rating, a foster parent, for instance, is at a point in this room. Very good identities are near the ceiling, and very bad identities are near the floor. Very powerful identities are near the front wall. Very powerless identities are near the back wall. Very active identities are near the right wall, while very quiet or inactive identities are near the left. The foster parent identity is located in this room, similar to the way a star is located in the cosmos, by its rating on all three dimensions. The meaning of any emotion (love directed to a foster parent) is also found in this three-dimensional EPA space (MacKinnon \& Keating, 1989). As a person moves through this space, the comparison continues; her emotional experience is determined by the absolute position in the room and by the direction of movement (Smith-Lovin \& Douglas, 1992).

Theoretical explanations are like paragraphs. Translation by explanations interprets deductive, propositional paragraphs, and inductive paragraphs. Affect con- 
trol theorists have deduced propositions about the basic characteristic of the relation between identity and emotions. Regarding intensity, ACT contends emotional intensity signals the degree to which an identity has been disconfirmed by an event (Francis, 2006). Specifically, the greater the degree of discordance between fundamental sentiments and impressions, the more intense is the arousal of emotion (Turner \& Stets, 2006). Regarding identity modifiers, Smith Lovin (1990) contends that the more stigma associated with an identity, the more negative the emotions. Additionally, the more power associated with the identity, the more likely the person experiences deep high-potency emotions, such as pride and fury, while the less powerful the positional identity, the more likely the person will experience emotions of impotency, such as fear, anxiety, and depression. Regarding situation variables, ACT theorists propose predictable relationships between situation type and the type of emotional arousal (Smith-Lovin, 1990). First, the greater the disagreement about the definition of a situation (I see myself as therapist, a client sees me as boyfriend), the more likely there will be a disconfirmation of identities and the arousal of emotions. Second, the greater the difference between cultural backgrounds, the greater the likelihood of emotional arousal due to disconfirmation of event elements. Finally, the more identities that a person enacts in a situation, the more likely that identity conflict will occur, some identities in the identity set will not be confirmed, and, consequently, the greater certainty of emotional arousal.

ACT theorists have theorized about and studied emotional displays (Heise, 1989, 2007). People judge each other on the basis of observable conduct, including the expressive signaling of subjective emotional states. The more that the actor displays culturally appropriate affect, the greater the likelihood that the actor's status will be enhanced. Conversely, the more often the actor displays inappropriate affect, the more likely the actor will be stigmatized. The proposal has been extended to courtroom judgments of culpability and punishment. A malicious action accompanied by emotions indicating remorse and guilt helps an actor more than malicious action accompanied by displays of satisfaction or glee (Smith-Lovin \& Robinson 2006).

In an example of intertextuality, ACT theorists have referred to Hochschild's (1983) work on emotion norms and work (Heise \& Calhan, 1995). Emotion norms prescribe a range of permitted feelings, given particular circumstances. Persons engage in emotion work in an effort to bring automatic emotional reactions in line with cultural expectations. Based on a study of college students, several propositions emerged. Influential people can more successfully shape emotional norms to reflect their ideological preferences and create resources for personal gain than minimally influential people. Moreover, there is a relationship between gender and emotions. Gender has minimal effect on the emotion norms that actors associate with particular events. However, women are more likely to report negative emotions, embarrassment, and vulnerability to events than men. Finally, the greater the discrepancy between emotion norms and emotion reactions (and the researchers found discrepancies in almost $50 \%$ of the 128 events), the greater the demands for emotion work, such as masking or overriding reactions. 
Thanks to the ACT theoretical research program, there are testable propositions about specific emotions. For ambivalence, Smith-Lovin (2003) proposes that, if an actor occupies more that one identity simultaneously and experiences events from these multiple perspectives, it is likely that events will evoke a mixture of emotions and the feeling of ambivalence. For example, an action supporting the identity of "judge" might produce negative deflection on the evaluation (likeability) and positive deflection on the potency dimensions of "women," resulting in a troubling mixture of feelings. In the case of anger as related to justice considerations, Scher and Heise (1993) propose that the more often a person who is involved in a transaction feels anger and the emotion is not ameliorated, the more likely that he will decide that the transaction is unjust. This relationship is conditioned by justice importance. so that the more one cares about justice, the greater the emotional impact of an incongruence. Regarding depression, McKinnon and Goulborne (2006) propose a relationship between the emotion and perceptions of one's situational identity. Those events that create transient identity impressions with a negative valence on evaluation, potency, and activity produce bad, powerless, and unlively emotions; that is, depression. Furthermore, people who often enact social disvalued identities more typically experience depressed emotions compared to those who enact non-stigmatized identities. Finally, the more that positively evaluated identities are disconfirmed in interaction or stigmatized identities are continuously confirmed, the more likely depression will become an enduring mood. For surprise, Smith-Lovin (2003) suggests that the greater the disconfirmation's unexpectedness, the more the emotional experience of surprise, unreality, and confusion. Regarding stress, to the extent that a deflection is experienced psychologically as a sense that the world is unpredictable, not right, or disturbing, the greater the likelihood of stress. Smith-Lovin adds that the more frequent the experience of stress, the greater the probability that the disconfirmed actor will attempt to leave the interaction. Affect control theorists build on each other's work. Empirical studies translating these propositions into testable format and seeking verification are likely to follow.

Theoretical translation must also consider inductive work identifying processes, typologies, or themes. Affect control theorists explain the typical social process, an encounter, inductively (Francis, 2006; Scher \& Heise, 1993). First, the actors comprehend and define the social situation (a work meeting in an agency's office). Second, the actors anticipate, perceive, and interpret the elements of the event: identities, objects of actors' behavior, behaviors, and setting features. Third, the actors behave to confirm their pre-existing sentiments about these elements. Fourth, the actors appraise behavioral and emotional displays to determine how each participant is faring in the social interaction (good, potent, lively, or otherwise). Fifth, when unanticipated events occur (a senior social worker harshly criticizes a novice worker), the actors attempt to create subsequent events that fit within their frame of expected behaviors and confirm fundamental sentiments (other workers help the manager show consideration or workers redefine the manager's identity to be inept or cruel). The balancing or restorative actions for failed event confirmations can be one of three types. An actor can reconceptualize the role behavior of self or other, leaving identities intact: the supervisor is teaching a lesson not criticizing. An actor can reconceptualize the setting: the supervisor is 
critical, because it is a personnel evaluative session. An actor can reconceptualize the other's identity by adding a modifier: a mood, trait, or moral condition (senior social worker is grumpy, arrogant, unprofessional), or by assigning a new identity (the supervisor is a corporate hatchet man initiating a dismissal process).

\section{TRANSLATION FOR THEORY APPRAISAL}

Semiotics can assist us in appraising the ACT and identifying its strengths and limitations. Three types of standards must be used: universal standards (parsimony, testability, explanatory power, scope, predictive power, and so on), social work standards (strengths orientation, appreciation of social justice, sensitivity to diversity, biopsychosocial explanations, ethical and values integrity, attention to varied size-systems and life stages, and empirical support), and semiotic standards (degree of articulation of theory elements and their relationships, communicability) (Forte, 2006).

I will illustrate each. First, ACT is a parsimonious theory. "ACT = predictions for ANBOS based on EPA profiles" is my summary of the theory's ingredients. ACT theorizes that "humans try to manage experiences so that immediate feelings about people, actions, and settings affirm long-term sentiments" (Heise, in MacKinnon, 1994, p. xi). Each event is composed of predictable elements: an actor (A) performing an act, including nonverbal $(\mathrm{N})$ and verbal behavior $(\mathrm{B})$ on an object $(\mathrm{O})$ in a setting $(\mathrm{S})$. The EPA profile summarizes the fundamental sentiment on three dimensions (Francis, 2006). If we know the EPA for four of the five elements of an event, the affect control theorists can predict the sentiments for the fifth element. All ACT's theoretical models, middle-range theories, theoretical elaborations, and applications build on this foundation. This single integrated theory explains much about social life, the normal social actor, and what motivates and shapes behavior (Robinson \& Smith-Lovin, 2006).

Second, from the perspective of social work's preference for biopsychosocial theories, ACT is lacking. The theory gives minimal attention to the biological processes central to emotion and overemphasizes situational cues, cultural norms, and social structures (Turner \& Stets, 2006). Affect control theorists have responded to social work's appreciation for diversity. Meaning universally involves three dimensions. This has been confirmed in studies of more than 20 cultures (Robinson \& Smith-Lovin, 2006), and the affect control process applies cross-culturally. All people use the same psychological processes, but cultural dictionaries (the specific meanings for identities, emotions, behaviors, and settings) vary. Within a culture, there is consensus among persons integrated into the culture about the meanings of event elements. Cultures tend to be fairly stable in sentiments, too (Heise, 2007). But, there are identifiable variations across cultures and subcultures in the particular EPAS for event elements. By comparing the fundamental sentiments of more than six countries, affect control theorists have documented how members of different cultures perceive elements of events differently (Robinson \& Smith-Lovin, 2006). Robinson and Smith-Lovin also review how ACT documents the specific dictionaries endorsed by males, females, and by subcultural groups, including Internet networks, gay members of a religious community, people aligned with different political ideologies, psychiatric patients, and Alcoholics Anonymous 
members. Extending the ACT knowledge base to increase understanding of diverse groups is a priority of the theoretical research program.

ACT meets that standard of scientific rigor. Fararo (1997), one of sociology's theory experts, regards ACT as one of the "best developed empirically applicable models" (Fararo, in Heise, 2007, p. 4). Its measurement tools and mathematical model of meaning prediction have been carefully developed, tested, and improved continuously (Francis, 2006). Many of the theory's basic assertions and predictions have been validated. Heise and Weir (1999), for example, compared ACT predictions about emotions with what people reported they will experience in imagined situations. Their findings generally confirmed the theory. The emotions experienced were close to those predicted by ACT. Emotions predicted to be unlikely by ACT were rarely experienced. Lively and Heise's two studies (2004) of 2,904 English speakers supported the ACT assertion of the three-dimensional structure of emotions and ACT propositions about the intentional transformation of emotions and the specific pathways for change.

Third, theories are semiotic resources for communicating and meeting challenges encountered by theory users. ACT successfully carries out theory's communicative functions (Heise, 2007; Scher \& Heise, 1993). It describes well. EPA profiles have been collected as large data sets for numerous countries and subcultures. It predicts behavior. Interact, ACT computer software, can be used to predict the unknown element of an event, based on known elements. It has explanatory power. Act provides theoretical models to explain health outcomes, social movement transformations, support group dynamics, courtroom processes, and many other social experiences. It has reference use and serves as a highly developed and specialized language for collaborative inquiry by large a group of scientists. Affect control theorists have done an excellent job of transforming the theory into communicable form. Translation has been relatively easy. Root metaphors and propositions are explicitly identified. Concepts are defined conceptually and operationally. Historical exemplars and classical texts have been identified. The theory has even been presented in a comprehensive, plain English format (Heise, 2007).

\section{TRANSLATION FOR APPLICATION}

Scientists and social workers often use visual aids and words to depict highlighted aspects of a theory as a theoretical or practice model (Forte, 2006). Francis (1997) carried out a participant observational study of two different support groups: one for divorcees (spiritually-oriented) and one for bereaved persons. Based on her research and grounded theory method, Francis developed a useful ACT conceptual model of mutual aid processes. Support groups are focused on emotions and emotional behavior and are directed towards helping members with "interpersonal emotion management" (p. 153). Group leaders and members work to "shape, work, or manage emotions" (p. 153). Her model includes the following components. Each support group identified three actors with identities of consequence: self-ex-God for the divorce group. The group leader facilitated a process of redefining the meanings for each of these identities and for the traumatic event during three phases. First, the worker helped members examine their initial self-definitions, generally an EPA characterizing self as bad, weak, and inactive. The coun- 
selor challenged the negative self-label, argued that the sufferer is a good person with a "bad experience," and promised that the negative identities (failure, victim) and emotions (powerlessness, stunned, futility) resulting from the deep deflection could be resolved. In the second phase, the leader and members embrace new self-definitions. In the divorce group, the sufferer is redefined as good, strong, and active. The ex-spouse is defined as bad, weak, and active, and God is introduced as a resource. In the third phase, there is further definitional and emotion work. The sufferer's identity as good, strong, and active is reaffirmed, and God is identified as a very good, very strong, and very active ally in the healing process. With changes of identity meanings, new emotions are experienced, labeled, and discussed. These include determination, rejoicing, and trust. During the process of affective resocialization, members learned to redefine elements of the difficult event, reduce the event-caused deflection between fundamental sentiments and transient impressions, and replace negative identities with positive ones. Members confirmed each other's new identities, and these positive identities generated behaviors and emotions considered healthy by the leaders and members.

ACT also provides theoretical models relating identity, emotion, and interaction to health outcomes (Francis, 2003), showing how gay rights social movement leaders use emotion management techniques to transform members' emotions from fear, to anger, to pride (Britt \& Heise, 2000); diagramming the relationship between situational factors, emotional arousal, and cognitive deliberations about justice (Scher \& Heise, 1993); profiling a gay-friendly church and how it uses rituals to change the religious and gay identities of its members from stigmatized to positive meanings (Smith-Lovin \& Douglas, 1992); and identifying the conditions necessary to build "empathic solidarity" (Heise, 1998). These should be adapted for social work use.

A theoretical language must be translated from abstractions into strategies for practical use during the helping process (Forte, 2006). Specifically, a theory translator must interpret theoretical knowledge to direct every phase of the planned change process, including engagement, assessment, intervention, and evaluative activities. For the engagement phase, Francis (2006) hints at a root metaphor for "social worker," when she characterizes the support group leader as a facilitator of "assisted deflection resolution." Expanding on this kernel, we can imagine the ideal engagement of the client from the ACT perspective. The client has been deflected or set off course by a disconfirmation. The worker reaches out to identify the original course settings (EPA profiles on situational elements), communicate respect for and validation of these sentiments, and most importantly, convey the conviction that the client, with assistance, can again take control of affect and return to course settings (Francis, 2006). Imagery and concepts associated with guide and guidance, sailing and navigating following a disorienting storm, revalidation, and reconfirmation might embellish the root metaphor.

For the data gathering and assessment phases, ACT can be translated into the verbal and visual language of the eco-map. The focal system would be the actor enacting an identity in a social situation (MacKinnon, 1994). The identity can be described concisely with modifiers, including mood, trait, and moral characteristics, and the actor's behavior can be described with quantitative modifiers, includ- 
ing always, sometimes, or rarely. Heise (2007) has researched the range of possible focal identities. In the United States, actors can enact between 500 and 1000 identities. These are related to socioeconomic and work systems; kinship, political systems, and religious organizations; categorical memberships, such as age, sex, and race; ethnic and ancestral heritage other than race; global identities associated with personality traits; leisure time and hobbies, sexual orientation, and sexual style. The systems surrounding the focal system are social institutions. Institutions are "constellations of roles, identities, settings, and actions related to some elementary concern" (Heise, 2007, p. 28). Surrounding circles would then include the family (marriage, child care, and caregiving); the realm of sexual encounters; business (working at a job and buying and selling in markets); religion (organizations and divine beings); education at all levels; medical; legal (judicial and law enforcement); political (executive and electoral government at all levels); leisure, including travel and entertainment; military; and science. The total environment on the eco-map would be a society's entire social structure of institutions and identities. Connections between the focal actor and actors in various institutions involve identity-relevant actions. Positive connections are those that confirm the person's identity. Stressful or negative connections are those that disconfirm the identity (Smith-Lovin, 2003).

Theory-based assessment tool can also be built from the operational definitions of theory variables. The procedure for assessing evaluation-potency-activity profiles is based on Osgood's semantic differential research (Smith-Lovin \& Robinson, 2006). The procedures are clear and specific. The measurement approach can be used to assess identities, behaviors, objects, emotions, and settings, and the measurement procedure allows for adjustment for institutional domains and cultures. Each of the three dimensions is placed on a nine-point scale from the extremes. Evaluation, for example, uses a good/bad and positive/negative valence for its continuum (Scher \& Heise, 1993). The measurement procedure can be used in either paper-and-pencil format or as a computerized software program. A social worker might collect information on the meanings assigned by a client to all elements of an event, then compare this to the shared cultural meanings documented by affect control theorists. EPA profiles can also be used to assess current meanings and predict likely emotions or behaviors based on those meanings. ACT researchers have used the measurement procedures in dozens of studies. Unfortunately, there is no example of its use for helping purposes.

During the intervention phase, social workers might use ACT by applying its ifthen statements (Forte, 2006). The "if" part of the clause refers to personal and membership processes and structures where intervention is possible, and the "then" part refers to the probable outcomes of intervention for the quality of biopsychosocial functioning. Several translations follow. Regarding consistent disconfirmation, to the degree that a person is in a relationship characterized by the repeated disconfirmation of a positive identity (wife abused by a spouse), then depression will result. If the worker can help the person avoid or exit the situation, or if the worker can help the person participate in support groups where others validate positively evaluated, powerful, and lively identities, then there will be a reduction of deflection and emotions maximally distant from depression will be 
generated (MacKinnon \& Goulbourne, 2006). Regarding event characteristics, if the client is in a situation where expectations are violated and identities are disconfirmed, then socially undesirable emotions will be evoked (Heise, 2007). If the worker can implement a new event that replaces the unwanted emotion with a different emotion, or if the worker can help the client reinterpret the past event so that the emotion produced by the original interpretation is replaced by another emotion, then equilibrium will be achieved again (Heise \& O'Brien, 1993).

Translation by exemplar is another strategy for translating a theory for intervention purposes. This sense-making task requires the practitioner to imagine that he or she could ask a theory's exemplars for intervention advice and then use this advice to increase professional effectiveness. "What would David Heise and other affect control theorists do in this situation?" Unfortunately, the ACT literature provides few clues about how David Heise or any affect control theorist or researcher would engage in service or answer specific social work practice questions. One piece of advice comes from Lively and Heise (2004). Social workers should use ACT-based emotional shortcuts to help people move between positive and negative emotions.

\section{CONCLUSION: FUTURE WORK ON ACT AND SEMIOTIC TRANSLATION}

Social workers need theory translation for many reasons. Theories are often stated in obscure, unclear ways. Theorists use abstract, general terms that are hard to apply to the particulars of social work situations. Some theorists use ill-defined terms. Some use well-defined terms in unusual ways. Some use specialized terms that are very unlike words from natural languages. Theory translation is necessary, because there are so many theoretical languages and because the major theoretical languages are alive and changing continually. Theory translation can make communication possible between theory specialists (theorists), theory users (practitioners), theory testers (researchers), and the beneficiaries of theories (clients). Theory translation is necessary, because theoretical explanations must be adjusted for different contexts, including team meetings, sessions with clients, and discussions with family members. Translators who specialize in particular languages can help (Forte, 2006).

This article has been an exercise in theory translation. A semiotic metatheory was offered as a framework for understanding science and scientific theories. Affect control theorists ponder and investigate how actors, identities, actions, objects, emotions, and social settings are interrelated during social interaction. ACT has been interpreted in terms of its value in increasing our understanding of human behavior in the social environment, for its suitability to social work, and for its applicability. A variety of theory translation tools were used to translate ACT technical words into simpler language, ACT displays into words and ACT's interactionist language into the language and imagery of ecosystems theory.

Semiotic metatheorizing about ACT can be taken further. ACT uses the language of mathematics in its formal theory statements and prediction equations. I am not fluent in mathematics and am unable to translate these statements. Additionally, semioticians are concerned about "polysemy," the notion that any sign has multiple meanings and translators focus selectively and differently on the sign's mean- 
ing (Colapietro, 1993). My translation needs to be compared to that of theory experts. Finally, this paper has addressed the linguistic aspects of text analysis. Future communication-oriented theory appraisal should consider the ideological positions embedded in ACT texts and ACT's use of rhetoric to persuade readers.

This article represents an exploratory effort in the use of semiotics as a metatheory for translating theories. At this point, social work lacks a semiotic pedagogy for theory learning. Pedagogical principles, lessons, drills, and vocabulary tests based on this foundation have not been developed. Additionally, the semiotic translation skills for the mastery of ACT and other theories have not yet been codified. Further work might specify the principles and procedures associated with profiling exemplars, creating and reading theoretical models, comparing target and source domains to generate root metaphors, deconstructing a theory's architectonic, and identifying plain English equivalents for theoretical keywords.

While ACT provides useful root metaphors for the person and the environment, its theory-based root metaphor for the social work helper is too sketchy. Social work has not yet established evidence-based links between particular theoretical languages, such as ACT, and the fields and practice settings in which the languages are most useful. Affect control theorists make some claims about applicable situations. Social workers need to validate these claims. Conceptual models developed by ACT to guide emotion work are based on studies of the planned change activities conducted by non-ACT practitioners. These conceptual models have not been based on the direct application of ACT to practice situations. Social workers must develop ACT models related to our practice efforts and begin to incorporate ACT concepts, propositions, models, and findings into our own personal practice models.

A translator specializes in providing equivalent terms in a target language for terms in a source language (Sarukkai, 2002). The translator serves practitioners who are eager to learn new theoretical languages by making sense out of some text, discourse, or other semiotic system (Colapietro, 1993). Science is a sign system. Scientists use symbols, icons, and indexes that seem like an unbreakable code and a foreign language. Translators can help social workers develop the theoretical competencies necessary for multi-modal literacy and fluency so that we can better understand, appraise, and use powerful theories like Affect Control Theory.

\section{References}

Allen-Meares, P., Hudgins, C.A., Engberg, M.E., \& Lessnau, B. (2005). Using a collaboratory model to translate social work research into practice and policy. Research on Social Work Practice, 15(1), 29-40.

Averett, C., \& Heise, D.R. (1987). Modified social identities: Amalgamations, attributions, and emotions. Journal of Mathematical Sociology, 13(1-2), 103-132.

Bloom, M. (1975). The paradox of helping: Introduction to the philosophy of scientific practice. New York: John Wiley and Sons.

Bloom, M., Wood, K, \& Chambon, A. (1991). The six languages of social work. Social Work, 36(6), 530-534. 
Britt, L., \& Heise, D.R. (2000). From shame to pride in identity politics. In S. Stryker, T.J. Owens, \& R.W. White (Eds.), Self, identity, and social movements (pp. 252-268). Minneapolis: University of Minneapolis Press.

Colapietro, V.M. (1993). Glossary of semiotics. New York: Paragon House.

Danesi, M. (1998). Sign, thought, and culture: A basic course in semiotics. Toronto, Ontario: Canadian Scholar's Press.

Forte, J.A. (2001). Theories for practice: Symbolic interactionist translations. University Press of America, Lanham, Maryland.

Forte, J.A. (2002). Mead, contemporary metatheory, and twenty-first-century interdisciplinary team work, Sociological Practice: A Journal of Clinical and Applied Sociology, 4(4), 315-334.

Forte, J.A. (2006). Human behavior and the social environment: Models, metaphors, and maps for applying theoretical perspectives to practice. Belmont, CA: Thomson Brooks/Cole.

Francis, C.A. (2006). Introduction to affect control theory. In K.A. McClelland \& T.J Fararo (Eds.), Purpose, meaning, and action: Control systems theories in sociology (pp. 139-161). New York: Palgrave MacMillan.

Francis, L.E. (1997). Ideology and interpersonal emotion management: Redefining identity in two support groups. Social Psychology Quarterly, 60(2), 153-171.

Francis, L.E. (2003). Feeling good, feeling well: Identity, emotion, and health. In P.J. Burke, T.J. Owens, R.T Serpe, \& P.A. Thoits (Eds.), Advances in identity theory and research (pp. 123-134). New York: Kluwer Academic/Plenum.

Halliday, M.A.K. (1978). Language as social semiotic: The social interpretation of Language and meaning. Baltimore, MD: University Park Press.

Heise, D.R. (1979). Understanding events: Affect and the construction of social action. New York: Cambridge University Press.

Heise, D.R. (1989). Effects of emotion displays on social identification. Social Psychology Quarterly, 52(1), 10-21.

Heise, D.R. (1998). Conditions for empathic solidarity. In P. Doreian \& T. Fararo (Eds.), The problem of solidarity: Theories and models (pp. 197-211). Amsterdam: Gordon and Breach.

Heise, D.R. (2007). Expressive order: Confirming sentiments in social actions. New York: Springer.

Heise, D.R., \& Calhan, C. (1995). Emotion norms in interpersonal events. Social Psychology Quarterly, 58(4), 223-240.

Heise, D.R., \& O’Brien, J. (1993). Emotion expression in groups. In M. Lewis \& J.M. Haviland (Eds.), Handbook of emotion (pp. 489-497). New York: The Guilford Press.

Heise, D.R., \& Weir, B. (1999). A test of symbolic interactionist predictions about emotions in imagined situations. Symbolic Interaction, 22(2), 139-161.

Hochschild, A.R. (1983). The managed heart: The commercialization of human feeling. Berkeley and Los Angeles: University of California Press.

Hodge, R., \& Kress, G. (1988). Social semiotics. Ithaca, NY: Cornell University Press.

Hudgins, C.A., \& Allen-Meares, P. (2000). Translation research: A new solution to an old problem. Journal of Social Work Education, 36(1), 2-5.

Jacobson, R. (1965). On linguistic aspects of translation. In R.A. Brower (Ed.), On translation (pp. 223-239). New York: Oxford University Press.

Lively, K.J., \& Heise, D.R. (2004). Sociological realms of emotional experience. American Journal of Sociology, 109(5), 1109-1136.

MacKinnon, N.J. (1994). Symbolic interactionism as affect control. Albany, NY: State University of New York Press.

MacKinnon, N.J., \& Goulbourne, M.M. (2006). The affect control theory of emotions: The case of depression. In K.A. McClelland \& T.J Fararo (Eds.), Purpose, meaning, and action: Control systems theories in sociology (pp. 237-266). New York: Palgrave MacMillan. 
MacKinnon, N.J., \& Keating, L.J. (1989). The structure of emotion: Canada-United States comparison. Social Psychology Quarterly, 52(1), 70-83.

Morris, C. (1946). The significance of the unity of science movement. Philosophy and Phenomenological Research, 6(4), 508-515.

Osimo, B. (2002). On psychological aspects of translation. Sign System Studies, 30(2), 607-627.

Rashotte, L.S. (2002). Incorporating nonverbal behaviors into affect control theory. Electronic Journal of Sociology, 6(3).

Ritzer, G. (1991). Metatheorizing in sociology. Lexington, MA: Lexington Books.

Robinson, D.T., \& Smith-Lovin, L. (2006). Affect control theory. In P.J. Burke (Ed.), Contemporary social psychological theories (pp. 137-154). Stanford, CA: Stanford Social Sciences.

Sarukkai, S. (2002). Translating the world: Science and language. Lanham, MD: University Press of America.

Scher, S.J., \& Heise, D.R. (1993). Affect and the perception of injustice. Advances in Group Processes, 10, 223252.

Smith, H.W., \& MiowLin, Y. (2006). Guilty Americans and shameful Japanese? An affect control theory test of Benedict's thesis. In K.A. McClelland \& T.J Fararo (Eds.), Purpose, meaning, and action: Control systems theories in sociology (pp. 163-188). New York: Palgrave MacMillan.

Smith-Lovin, L. (1987). The affective control of events within settings. Journal of Mathematical Sociology, $13,71-101$

Smith-Lovin, L. (1989). Sentiment, affect, and emotion. Social Psychology Quarterly, 52(1), x-xii.

Smith-Lovin, L. (1990). Emotion as the confirmation and disconfirmation of identity: An affect control model. In T.D. Kemper (Ed.), Research agendas in the sociology of emotion (pp. 238-270). Albany, NY: State University of New York Press.

Smith-Lovin, L. (1995). The sociology of affect and emotion. In K.S. Cook, G.A. Fine, \& J.S. House (Eds.). Sociological perspectives on social psychology (pp. 118-148). Boston: Allyn \& Bacon.

Smith-Lovin, L. (1999). Introduction of David R. Heise: Recipient of the 1998 Cooley-Mead award. Social Psychology Quarterly, 62(1), 1-3.

Smith-Lovin, L. (2003). Self, identity, and interaction in an ecology of identities. In P.J. Burke, J. Owens, R.T. Serpe, \& P.A. Thoits (Eds.), Advances in identity theory and research (pp. 167-178). New York: Kluwer Academic/Plenum.

Smith-Lovin, L., \& Douglas, W. (1992). An affect control analysis of two religious groups. In D.D. Franks (Ed.), Social perspectives on emotion. Volume 1 (pp. 217-247). Greenwich, CT: JAI Press.

Smith-Lovin, L., \& Heise, D.R. (1988). Analyzing social interaction: Advances in affect control theory. New York: Gordon and Breach.

Smith-Lovin, L. \& Robinson, D.T. (2006). Control theories of identity, action, and emotion: In search of testable differences between affect control theory and identity control theory. In K.A. McClelland \& T.J Fararo (Eds.), Purpose, meaning, and action: Control systems theories in sociology (pp. 163-188). New York: Palgrave MacMillan.

Turner, J.H., \& Stets, J.E. (2005). The sociology of emotions. New York: Cambridge University Press.

Watson, W. 1985. The architectonics of meaning: Foundations of the new pluralism. Albany, NY: State University of New York Press.

Zhao, S. (2001). Metatheorizing in sociology. In G. Ritzer \& B. Smart (Eds.), in Handbook of social theory (pp. 386-394). London: Sage.

\section{Author's Note:}

Address correspondence to: Professor James Forte, Department of Social Work, 1101 Camden Avenue, Salisbury, Maryland, 21801, USA. e-mail: jaforte@salisbury.edu or jamesforte@mac.com http://web.mac.com/jamesforte/iWeb/ 